\title{
Optical injection dynamics of frequency combs
}

\author{
Yaya DOUMBIA, Tushar MALICA, Delphine WOLFERSBERGER, Krassimir PANAJOTOV and \\ Marc SCIAMANNA
}

\begin{abstract}
We analyze the nonlinear dynamics of a semiconductor laser with optical injection from a frequency comb. When varying both the injection parameters (detuning and injection ratio), and the comb properties (number of comb lines and comb spacing), we identify and select several dynamics including (i) injection locking with selective amplification of the comb line that shows the smallest detuning from the injected laser, (ii) unlocked time-periodic dynamics that correspond to a new frequency comb with a broadened optical spectrum thereby sharpening the pulse generated at the injected laser output, and (iii) unlocked chaotic dynamics.
\end{abstract}

Keywords: Semiconductor lasers, nonlinear dynamics, optical injection, frequency comb.

The injection locking (IL) properties of semiconductor lasers have been analyzed since more than forty years: first, as a way to control the coherence properties of the injected laser, but more recently, as a technique to tailor specific nonlinear dynamics including optical chaos [1,2], time-periodic self-pulsation [3], and also dissipative solitons in large aperture laser diodes $[4,5]$. These nonlinear dynamics found applications in various fields such as physical security based on optical chaos [6,7], optical sensing [8] and radio-over fiber communications [9-11]. It is known that a multifrequency injected laser, unlike the case of a single mode injected laser diode, brings additional nonlinear dynamics and examples of which can be found in injection experiments exploring polarization dynamics in VCSELs [12-14], longitudinal mode dynamics in quantum dot lasers $[3,15]$, and the so-called two-colour laser diodes [16]. Much less studied is the dynamics of a single mode laser diode injected by a multi-frequency laser diode. This configuration has raised recent interest within the context of optical frequency combs, i.e. a coherent set of optical modes being modelocked and producing possibly very short pulses in the laser output [17-19]. Recent experimental and theoretical work have considered optical injection of such frequency combs as a way to selectively amplify individual comb lines and therefore, tailor the comb properties [20-23]. In addition, the question of how optical injection induced nonlinear dynamics impacts the comb properties in the injected laser is of great fundamental interest and has not been addressed so far.

In this Letter, we make an in-depth analysis of singlemode semiconductor laser dynamics induced by optical injection of a frequency comb by varying both the injection parameters and the comb properties. Besides the IL and the selective amplification of the comb line with the smallest detuning from the injected lines that has been reported in several recent experiments $[22,23]$, we reveal a large set of nonlinear dynamics that bifurcate from the IL solution. Of particular interest is the onset of a new time-periodic dynamics in the unlocking regime that extends the injection combs lines to a much broader range of optical frequencies, hence, also significantly sharpening the pulse in the injected laser output. The number of the resulting comb lines can be tailored by varying the injection parameters and the initial comb properties (number of lines and the comb spacing). The laser system is modelled with the equations from Ref. [24] adapted to injection from a comb. The complex electrical field of the injected comb can be written as:

$$
E_{M}(t)=\sum_{j} E_{j}(t) e^{i\left(2 \pi \nu_{j} t+\varphi\right)},
$$

where $E_{j}(t)$ is the amplitude of the j-th comb-line, $\nu_{j}$ is the frequencie of the $\mathrm{j}$-th comb-line, $\varphi$ is the initial phase of each comb-line. We set the initial phase of each comb-line to 0 and we suppose that the comb lines have the sames amplitudes, $E_{i n j}$. By doing the same calculation as in [24], the injected laser's equations can be written as:

$$
\begin{gathered}
\dot{E}(t)=\frac{1}{2} G_{N}\left(N(t)-N_{t h}\right) E(t)+E_{i n j} \sum_{j} \cos \left(2 \pi \Delta \nu_{j} t-\phi(t)\right) \\
\dot{\Phi}(t)=\frac{1}{2} \alpha G_{N}\left(N(t)-N_{t h}\right)+\frac{E_{i n j}}{E(t)} \sum_{j} \sin \left(2 \pi \Delta \nu_{j} t-\phi(t)\right) \\
\dot{N}(t)=R_{p}-\frac{N(t)}{\tau_{s}}-G_{N}\left(N(t)-N_{t h}\right) E(t)^{2}-\frac{E(t)^{2}}{\tau_{p}}
\end{gathered}
$$

In these equations, $\Delta \nu_{j}$ is the detuning, $N_{t h}$ is the threshold carrier density, $\alpha$ is the linewidth enhancement, $G_{N}$ is the differential gain, $R_{p}$ is the pump rate and $\tau_{s}$ is the carrier lifetime. In the following, we shall use $\kappa$ for the injection ratio with $\kappa=\frac{E_{i n j}}{E_{0}}$, where $E_{0}$ is the field amplitude of the injected laser without optical injection. The semicondutor 
laser parameters are taken as $\alpha=5, G_{N}=7.9 \times 10^{-13}$ $m^{3} s^{-1}, N_{t h}=2.91924 \times 10^{24} \mathrm{~m}^{-3}, \quad \tau_{s}=2 \times 10^{-9} \mathrm{~s}$, $\tau_{p}=2 \times 10^{-12} \mathrm{~s}$ and $R_{p}=1.2 R_{t h}$ with the pump rate at threshold $R_{t h}=1.8 \times 10^{33} \mathrm{~s}^{-1}$. In equations (2)-(4) it is assumed that the bandwidth of the wavelength selective filter in the injected laser (gain bandwidth and cavity bandwidth) is much larger than the comb width, hence not preventing the new additional comb lines to be lasing as will be discussed here after. Rate equations (2)-(4) are integrated using the fourth-order Runge-Kutta method with a time step of $1.2 \mathrm{ps}$.

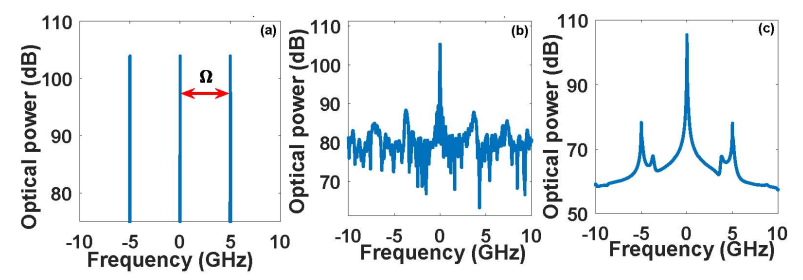

Figure 1: Optical spectra shown in (a) shows the injected comb for 3 comb lines, (b) free running injected laser and (c) injected laser under IL condition. $\Omega=5 \mathrm{GHz}$ is the comb spacing and $\Delta \nu=10$ $\mathrm{MHz}$ is the detuning.

Figure 1 shows optical spectra of the injected frequency comb [Fig. 1 (a)], the free running of the injected laser [Fig. 1(b)] and the injected laser under IL [Fig. 1 (c)]. The optical spectra in Figs. 1 (b) and (c) are obtained by discrete Fourier transform of the optical field from 80 ns long time-trace. IL is achieved in Fig. 1 (c) for $\Delta \nu=10$ $\mathrm{MHz}$ from the central comb line.

The frequency comb laser has 3 lines with equal amplitudes separated by $\Omega=\nu_{j+1}-\nu_{j}=5 \mathrm{GHz}$ and hence the injecting diode laser has modulated output power with pulse full-width at half maximum (FWHM) of 62.4 ps. The pulse repetition rate of $\Omega=5 \mathrm{GHz}$ correspond to the comb spacing. In IL condition Fig. 1 (c), the injected laser selectively amplifies the frequency comb line that is the closest to the injected laser frequency and simultaneously suppresses the side comb lines. The resulting injected laser output is not steady but modulated at a frequency matching $\Omega$. This selective amplification of a comb line under IL matches recent experimental observations $[22,23]$.

We next analyze the emergence of complex dynamics from the injection locked solution. For a fixed $\Delta \nu=0.2$ $\mathrm{GHz}$, and varying $\kappa$, the injected laser dynamics shows additional bifurcations from the IL solution as shown in Fig. 2 for 3 (top panels and 7 (bottom panels) of injected comb lines. Figures $2\left(a_{1}\right)$ and $\left(b_{1}\right)$ show wave mixing, i.e., the injected laser dynamics is intensity modulated as a result of nonlinear mixing between two frequencies, the detuning $\Delta \nu$ and $\Omega$.

Figures $2\left(a_{2}\right)$ and $\left(b_{2}\right)$ show IL for 3 and 7 comb lines injection. In both cases, the sidemode suppression ratio is the same (equal to $14 \mathrm{~dB}$ ). However, the selective amplification of the injected comb line is stronger for the 3-comb lines injection. The appearance of the additional comb lines is due to the nonlinear dynamics taking place in the injected laser, namely, the injection frequency comb leads to modulation of the carrier density at the frequency given by the spacing of the comb lines. Figures $2\left(a_{3}\right)$ and $\left(b_{3}\right)$ show two examples of chaotic dynamics. A close inspection of the corresponding power spectra shows that the chaos bandwidth of the 7 comb lines case $(10.57 \mathrm{GHz})$ is slightly larger than for the 3 comb lines case $(9.92 \mathrm{GHz})$, computed as in [25]. Figures $2\left(a_{4}\right)$ and $\left(b_{4}\right)$ correspond to the unlocked time-periodic dynamics with the injected laser output displaying a new frequency comb. We consider a new comb line when its amplitude lies above -30 $\mathrm{dB}$ from the maximum amplitude: we observe that the 7-comb lines injection leads to a wider optical spectrum with 29 lines involved in the comb rather than 17 lines for 3 -lines case. When further increasing $\kappa$, we find yet another comb with even broaded optical spectrum see Fig. $2\left(a_{5}\right)$ 3 -comb lines and Fig. $2\left(b_{5}\right)$ 7-comb line injection. The red dashed line in each optical spectrum indicates the injection locked frequency position, i.e., the frequency position of the injected central comb line. It is interesting to note that the unlocked comb is frequency shifted from the injection locked solution, with a frequency offset that increases with $\kappa$. The increase of $\kappa$ leads to a decrease of the average carrier density, yielding also more power in the injected laser and therefore to a redshift of the instantaneous frequency through equation (3). This explains the shift of the comb main line to the red when increasing $\kappa$.

The 3-comb lines and 7-comb lines injection cases differ by the range of injection parameters in which these new comb are observed. In Fig. 3, we map in detail the region of the time-periodic dynamics in the plane of the injection parameters. To this aim, We follow the maxima of the time series and compare the heights of the consecutive maxima for all the maxima lying above the mean. We consider a time series to be a comb when this difference is less than $0-2 \%$ of the maximum amplitude meaning that the dynamics is indeed time-periodic. The region shaded in blue corresponds to the time-periodic dynamics, i.e., either an injection locked, or an unlocked injected laser comb. The region shaded in red corresponds to any other unlocked dynamics. We are not interested here by the peculiar chaotic properties of the injected laser dynamics but, in the parameter range in which the injected laser shows an optical comb dynamics and the corresponding comb properties. Interestingly, the unlocked comb dynamics of the injected laser extends to a much broader range of injection parameters than the IL solution. Figures 3 (a)-(c) correspond to 3-comb lines injection with $\Omega$ equal to 3,5 and $10 \mathrm{GHz}$, respectively. Figures 3 (e)-(g) correspond to 7-comb lines injection with $\Omega$ equal to 3,5 and $10 \mathrm{GHz}$, respectively. The region around zero $\kappa$ is the IL region. We identify as many IL regions as the number of the comb lines, labelled 'IL' in the mapping. The IL region is limited when the ratio between the power of the comb line that shows the smallest detuning from the injected comb and the next strongest comb line is more than $10 \mathrm{~dB}$. The green points in the map- 

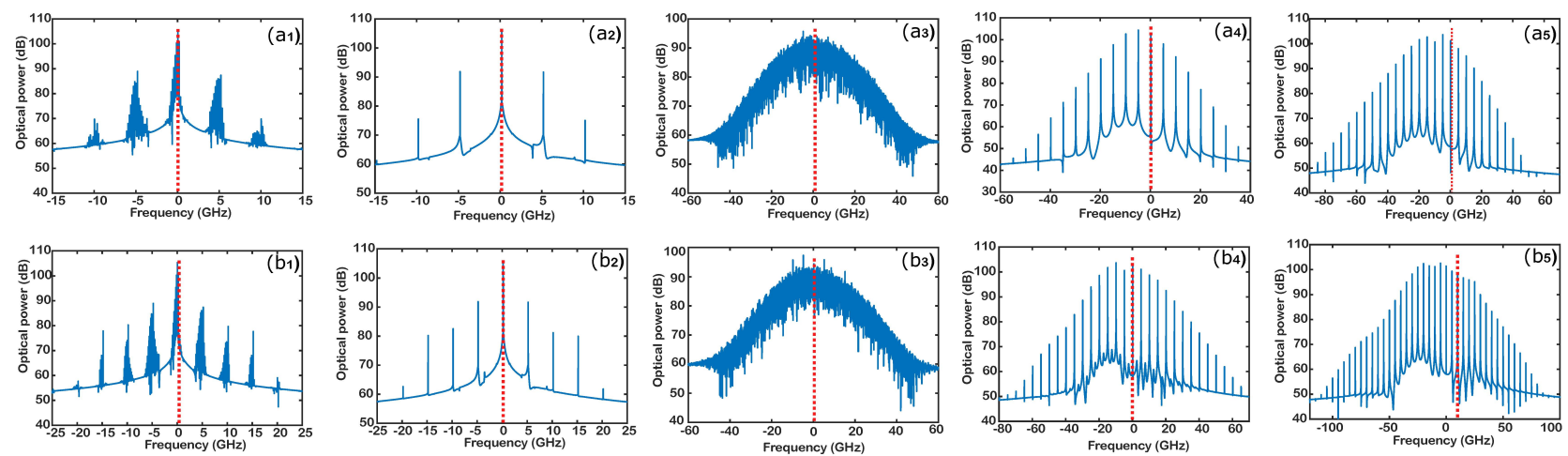

Figure 2: Optical spectra when varying the injection ratio $\kappa$.The top and bottom panels correspond to 3 and 7 comb line injection, respectively. All the figures are obtained with $\Delta \nu=0.2 \mathrm{GHz}$ except $b_{5}$ which is obtained with $\Delta \nu=10 \mathrm{GHz}$. Evolution of dynamics is as follows $\left(a_{1}\right)$ and $\left(b_{1}\right)$ wave mixing at $\kappa=0.006,\left(a_{2}\right)$ and $\left(b_{2}\right)$ injection locking at $\kappa=0.012,\left(a_{3}\right)$ and $\left(b_{3}\right)$ chaos at $\kappa=0.1,\left(a_{4}\right)$ and $\left(b_{4}\right)$ first unlocked time-periodic regime for 3 and 7 comb line at $\kappa=0.202$ and $\kappa=0.284$, respectively and $\left(a_{5}\right)$ and $\left(b_{5}\right)$ second unlocked time-periodic regime at $\kappa=0.6$. The red dashed line in each optical spectrum indicates the injection locked frequency position.
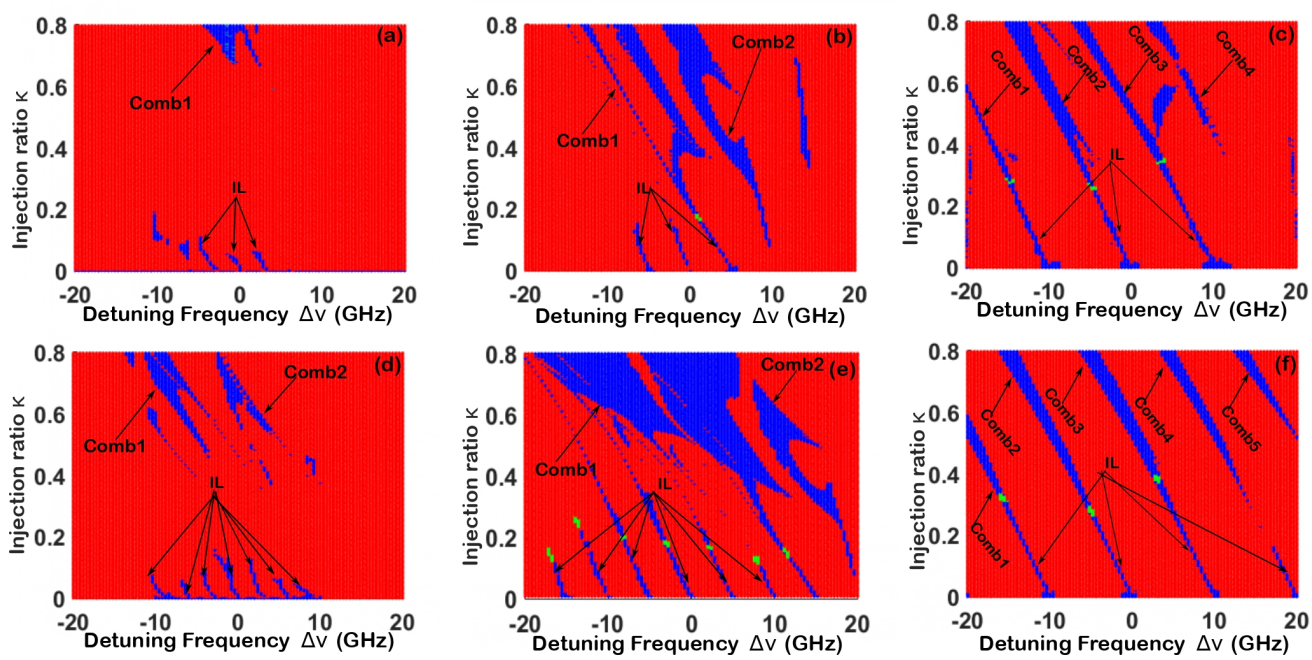

Figure 3: Numerical mapping of the semiconductor laser dynamics when subject to optical injection with frequency combs is shown by varying $\kappa$ as a function of detuning frequency for for comb spacing of 3, 5 and $10 \mathrm{GHz}$ respectively for (a)-(c) 3 comb lines injection and, (d)-(f)7 comb lines. Different regions are observed: Injection locking (IL), unlocked time-periodic dynamics (Comb1, Comb2, Comb3, Comb4 and Comb5). The green color corresponds to the limit of the injection locking region.

ping are the limit of the IL region. In the case of $\Omega=5 \mathrm{GHz}$, we observe two regions of unlocked time-periodic dynamics (see regions labelled, 'comb1' and 'comb2' in the mapping). The areas corresponding to the new comb extend both with $\kappa$ and with $\Delta \nu$. The new combs are observed for $\Omega$ ranging between below and up to several times relaxation oscillation frequency $(3.6 \mathrm{GHz})$. This confirms that the comb appearance is not restricted to injected comb spacing $\Omega$ close to the oscillation relaxation frequency. Tongue-like comb regions in the mapping (Fig. 3 (c) and (f)), are redishifting when increasing $\kappa$ and become more structured and narrower when increasing $\Omega$. We also notice that when increasing the number of the injected comb lines, the areas corresponding to the new comb extend more towards the negative detuning as $\kappa$ increases. As is made clear for example in Fig. 3(b) for $\Omega=5 \mathrm{GHz}$, the new comb connects to the IL solution, hence suggesting that this new comb indeed originates from the IL solution. We have checked that the transition from the IL to the comb is smooth with an increased power of the comb lines and the newly created equidistant frequency lines.

Tailoring the comb properties by varying the injection parameters is better seen in Fig. 4: we plot the pulse of the injected laser output when varying $\kappa$ within the parameter range corresponding to the unlocked injected laser comb (comb1 and comb2) of the Fig. 3 (b). In Fig. 4 (a), the blue and red curves correspond to the pulses in (comb1) and (comb2) region for $\kappa=0.35$ and $\kappa=0.6$ respectively with the same detuning $\Delta \nu=0.2 \mathrm{GHz}$. The pulse width decreases when $\kappa$ increases. In Fig. 4 (b) we show the number of comb lines of the injected laser versus number of lines of the injected comb. The new comb becomes wider with the number of injected comb lines. It is interesting to note that the new comb extends more when increasing the 

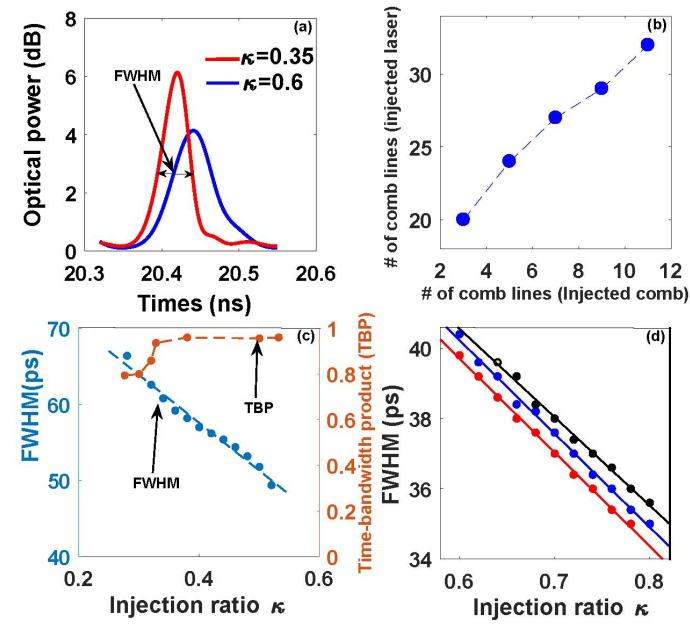

Figure 4: Control of comb properties for $\Omega=5 \mathrm{GHz}$ for 3 comb lines injection. (a) pulses in comb1 and comb2 for $\Delta \nu=0.2 \mathrm{GHz}$, $\kappa=0.35$ (red) and $\kappa=0.6$ (blue). (b) number of comb lines in the injected laser versus number of lines of the injected comb for $\Delta \nu=0$ $\mathrm{GHz}$ and $\kappa=0.8$. (c) FWHM of the comb1 on the left vertical axis and time-bandwidth product (TBP) on the right vertical axis when increasing $\kappa$. (d) same as (c) for the comb2 also comparing the cases of Fig. 3 (b) for $\Delta \nu=-0.7 \mathrm{GHz}$ (red), $\Delta \nu=0 \mathrm{GHz}$ (blue) and $\Delta \nu=0.7 \mathrm{GHz}$ (brown).

drive current of the injected laser. For example, in the case of Fig. 5 (a), when we increase the drive current of the injected laser by $15 \%$, the number of comb line goes from 21 to 27. In Fig. 4 (c) we show the pulse width on the left vertical axis for comb1 and the time-bandwidth product (TBP) on the right vertical axis when increasing $\kappa$. The TBP is calculated from the product between the FWHM and the spectral width at half maximum that is computed from the simulated optical spectra. The pulse width decreases with $\kappa$, but the TBP increases, suggesting that the pulse width is not only determined by the increase of spectral bandwidth, but also that there should be a dependency on the phase of the comb line as will be discussed here after. The TBP deteriorates as the pulses width decreases and reaches about twice the Fourier limit $\sim 0.44$ for a Gaussian shape pulse. Figure 4 (d) shows the pulse width for 3 detuning values of $0.7 \mathrm{GHz}, 0 \mathrm{GHz}$ and $-0.7 \mathrm{GHz}$ for the case of comb2. The pulse width is smaller for the negative $\Delta \nu$ than for the zero or positive $\Delta \nu$.

Our statement above on the phase of the comb lines is further supported by the analysis of Fig. 5. Figures 5 (a) and (b) show the optical spectra on the left vertical axis and the phase of each comb line on the right vertical axis. In both cases, we see that the comb lines have an almost constant phase difference between them. The linear fit of the phase difference versus frequency gets a little bit worst when increasing the number of comb lines as shown in Fig. 5 (c).

In summary, we show that it is possible to generate a broadened frequency comb using injection locking. We demonstrate this technique using a frequency comb as injected laser and single mode laser as injected laser. When

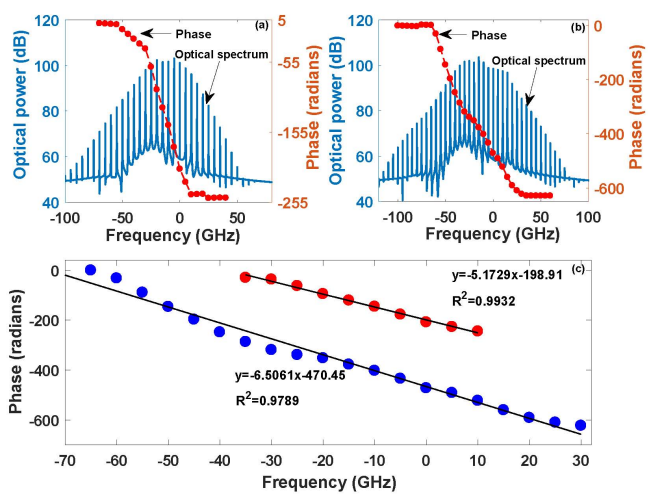

Figure 5: Optical spectra and phase for each comb line for $\Delta \nu=0$ $\mathrm{GHz}, \kappa=0.8$ and $\Omega=5 \mathrm{GHz}$. (a) and (b), optical spectra (left vertical axis ), phase (right vertical axis) for 3 and 7 comb line, respectively. (c) fitting of the linear part of the phase dependance for 3 comb lines (red) and 7 comb lines (blue)

increasing the injection ratio, the injected laser first shows wave mixing with its intensity modulated from the nonlinear mixing between the detuning frequency and a new frequency that depends on the comb mode spacing. Increasing the injection ratio, the injected laser gets locked with selective amplification of the comb mode with smallest detuning to the injected laser. Consecutively, more complex, including chaotic, dynamics is observed. Most importantly, new comb solutions take place for still increased injection ratio. The number of lines involved in the new comb solution, and therefore, the pulse width of the mode-locked injected laser dynamics is controlled by injection parameters, and by the number of the injected modes. In particular, the injected laser pulse width decreases when increasing the injection ratio and when moving the injected comb towards more negative detuning.

Funding. Chaire Photonique: Ministère de l'Enseignement Supérieur, de la Recherche et de l'Innovation; Région Grand-Est; Département Moselle; European Regional Development Fund (ERDF); Airbus GDI Simulation; CentraleSupélec; Fondation CentraleSupélec. Fondation Supélec; Metz Metropole, Fonds Wetenschappelijk Onderzoek (FWO) Vlaanderen Project No.G0E5819N.

\section{References}

[1] M. Sciamanna and K. A. Shore, "Physics and applications of laser diode chaos," Nature Photonics, vol. 9, no. 3, p. 151, 2015.

[2] S. Wieczorek, B. Krauskopf, T. B. Simpson, and D. Lenstra, "The dynamical complexity of optically injected semiconductor lasers," Physics Reports, vol. 416, no. 1-2, pp. 1-128, 2005. 
[3] L. Olejniczak, K. Panajotov, H. Thienpont, and M. Sciamanna, "Self-pulsations and excitability in optically injected quantum-dot lasers: Impact of the excited states and spontaneous emission noise," Physical Review $A$, vol. 82, no. 2, p. $023807,2010$.

[4] M. Marconi, J. Javaloyes, S. Barland, S. Balle, and M. Giudici, "Vectorial dissipative solitons in verticalcavity surface-emitting lasers with delays," Nature Photonics, vol. 9, no. 7, p. 450, 2015.

[5] E. Averlant, M. Tlidi, H. Thienpont, T. Ackemann, and K. Panajotov, "Vector cavity solitons in broad area vertical-cavity surface-emitting lasers," Scientific Reports, vol. 6, p. 20428, 2016.

[6] R. Sakuraba, K. Iwakawa, K. Kanno, and A. Uchida, "Tb/s physical random bit generation with bandwidthenhanced chaos in three-cascaded semiconductor lasers," Optics Express, vol. 23, no. 2, pp. 1470-1490, 2015.

[7] S. Sivaprakasam and K. A. Shore, "Demonstration of optical synchronization of chaotic external-cavity laser diodes," Optics Letters, vol. 24, no. 7, pp. 466468, 1999.

[8] W. W. Chow and S. Wieczorek, "Using chaos for remote sensing of laser radiation," Optics Express, vol. 17, no. 9, pp. 7491-7504, 2009.

[9] L.-C. Lin, S.-H. Liu, and F.-Y. Lin, "Stability of periodone (p1) oscillations generated by semiconductor lasers subject to optical injection or optical feedback," Optics Express, vol. 25, no. 21, pp. 25 523-25 532, 2017.

[10] R. Slavík, F. Parmigiani, J. Kakande, C. Lundström, M. Sjödin, P. A. Andrekson, R. Weerasuriya, S. Sygletos, A. D. Ellis, L. Grüner-Nielsen et al., "Alloptical phase and amplitude regenerator for nextgeneration telecommunications systems," Nature Photonics, vol. 4, no. 10, p. 690, 2010.

[11] E. Sooudi, J. O'Gorman, P. Gunning, A. D. Ellis, F. C. G. Gunning, and R. J. Manning, "Practical and costeffective high-fidelity optical carrier dissemination using coherent communication techniques," Optics express, vol. 23, no. 17, pp. 21678-21689, 2015.

[12] I. Gatare, M. Sciamanna, J. Buesa, H. Thienpont, and K. Panajotov, "Nonlinear dynamics accompanying polarization switching in vertical-cavity surfaceemitting lasers with orthogonal optical injection," Applied Physics Letters, vol. 88, no. 10, p. 101106, 2006.

[13] A. Valle, I. Gatare, K. Panajotov, and M. Sciamanna, "Transverse mode switching and locking in verticalcavity surface-emitting lasers subject to orthogonal optical injection," IEEE Journal of Quantum Electronics, vol. 43, no. 4, pp. 322-333, 2007.
[14] F. Denis-le Coarer, A. Quirce, Á. Valle, L. Pesquera, M. Sciamanna, H. Thienpont, and K. Panajotov, "Polarization dynamics induced by parallel optical injection in a single-mode vcsel," Optics Letters, vol. 42, no. 11, pp. 2130-2133, 2017.

[15] C. Wang, R. Raghunathan, K. Schires, S.-C. Chan, L. F. Lester, and F. Grillot, "Optically injected inas/gaas quantum dot laser for tunable photonic microwave generation," Optics Letters, vol. 41, no. 6, pp. 11531156, 2016.

[16] S. Osborne, P. Heinricht, N. Brandonisio, A. Amann, and S. O'Brien, "Wavelength switching dynamics of two-colour semiconductor lasers with optical injection and feedback," Semiconductor Science and Technology, vol. 27, no. 9, p. 094001, 2012.

[17] K. Saha, Y. Okawachi, B. Shim, J. S. Levy, R. Salem, A. R. Johnson, M. A. Foster, M. R. Lamont, M. Lipson, and A. L. Gaeta, "Modelocking and femtosecond pulse generation in chip-based frequency combs," Optics Express, vol. 21, no. 1, pp. 1335-1343, 2013.

[18] A. C. Bordonalli, M. J. Fice, and A. J. Seeds, Optics express, vol. 23, no. 2, pp. 1547-1557, 2015.

[19] D. S. Wu, R. Slavík, G. Marra, and D. J. Richardson, "Direct selection and amplification of individual narrowly spaced optical comb modes via injection locking: design and characterization," Journal of Lightwave Technology, vol. 31, no. 14, pp. 2287-2295, 2013.

[20] A. Gavrielides, "Comb injection and sidebands suppression," IEEE Journal of Quantum Electronics, vol. 50, no. 5, pp. 364-371, 2014.

[21] H. Moon, E. Kim, S. Park, and C. Park, "Selection and amplification of modes of an optical frequency comb using a femtosecond laser injection-locking technique," Applied Physics Letters, vol. 89, no. 18, p. 181110, 2006.

[22] D. S. Wu, D. J. Richardson, and R. Slavík, in Integrated Optics: Physics and Simulations, vol. 8781. International Society for Optics and Photonics, 2013, p. 87810J.

[23] K. J. Shortiss, M. Shayesteh, and F. H. Peters, Optical and Quantum Electronics, vol. 50, no. 1, p. 49, 2018.

[24] F. Mogensen, H. Olesen, and G. Jacobsen, "Locking conditions and stability properties for a semiconductor laser with external light injection," IEEE Journal of Quantum Electronics, vol. 21, no. 7, pp. 784-793, 1985.

[25] G. Bouchez, C.-H. Uy, B. Macias, D. Wolfersberger, and M. Sciamanna, "Wideband chaos from a laser diode with phase-conjugate feedback," Optics letters, vol. 44, no. 4, pp. 975-978, 2019. 